Relations industrielles

Industrial Relations

\title{
Index — volume 31 - 1976
}

Volume 31, numéro 4, 1976

URI : https://id.erudit.org/iderudit/028760ar

DOI : https://doi.org/10.7202/028760ar

Aller au sommaire du numéro

Éditeur(s)

Département des relations industrielles de l'Université Laval

ISSN

0034-379X (imprimé)

1703-8138 (numérique)

Découvrir la revue

Citer ce document

(1976). Index - volume 31 - 1976. Relations industrielles / Industrial Relations,

31(4), 699-709. https://doi.org/10.7202/028760ar

Tous droits réservés (C) Département des relations industrielles de l'Université Laval, 1976
Ce document est protégé par la loi sur le droit d'auteur. L’utilisation des services d'Érudit (y compris la reproduction) est assujettie à sa politique d'utilisation que vous pouvez consulter en ligne.

https://apropos.erudit.org/fr/usagers/politique-dutilisation/ 


\section{INDEX - VOL. 31 - 1976}

\section{Index alphabétique des articles}

Action ouvrière: analyse de cas, syndicalisation locale et, par L.H.J. Groulx,

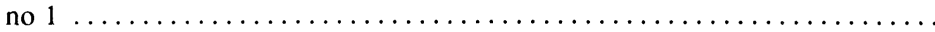

Activité économique, inflation et activité de grève, par J.M. Cousineau et

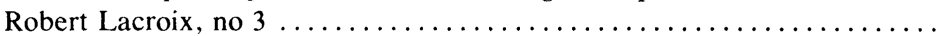

Accidents du travail, Management et prévention des - Les responsabilités des cadres à l'égard de la prévention, par Gérard Hébert, no $1 \ldots \ldots \ldots$.

Activité économique, le règlement des grèves et l', par R. Swidinsky, no $2 \ldots \ldots$ Advance Notice of Mass Activity: The Québec Experience, by Jean Sexton et

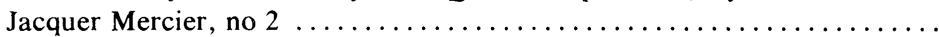

American Twist to a Canadian Approach, Public Sector Dispute Resolution:

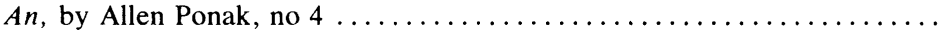

Americas, a Comparison of Wage Determination Methods in the, by Chris Jecchinis and Gérald Phillips, no $31 \ldots \ldots \ldots \ldots \ldots \ldots \ldots \ldots \ldots \ldots \ldots \ldots . \ldots \ldots$ Amériques, étude comparative des méthodes de fixation des salaires dans les, par Chris Jecchinis et Gérald Phillips, no $1 \ldots \ldots \ldots \ldots \ldots \ldots \ldots \ldots$ Anti-Inflation Board, the Canadian, by Claude Castonguay, no $4 \ldots \ldots \ldots \ldots$ Arbitration. A Model for the Study of Policy Change, Grievance, by Jeffrey

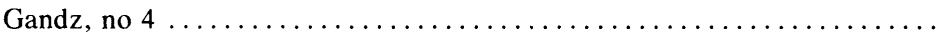

Arbitrage des griefs, $l$, par Jeffrey Gandz, no $4 \ldots \ldots \ldots \ldots \ldots \ldots \ldots \ldots$ Assurance-chômage, durée de chômage et offre de travail, par Dennis R.

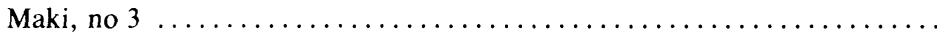

Attitudes patronales en matière de relations professionnelles: secteurs public et privé, par Mark J. Thompson et Larry F. Moore, no $3 \ldots \ldots \ldots \ldots \ldots$

Base: l'expérience d'Israël, structure syndicale et révolte de la, par Abraham

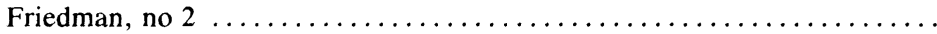

Blue Collar, Company Satisfaction, Company Commitment and Work Involvement: An Empirical Examination of Blue Collar, by V. Baba et M. Jamal,

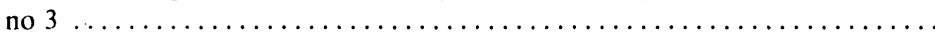

British Columbia's Construction Industry, A Note on Industrial Conflict in,

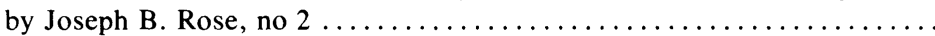

Cadres à l'égard de la prévention, Management et prévention des accidents

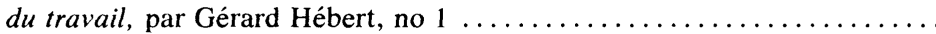

Canada, The Impact of Inflation on Strike Activity in, by Douglas A. Smith,

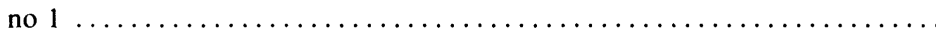

Canada: A Comment, Trade Union Growth in, by George Bain and F. Elsheikh,

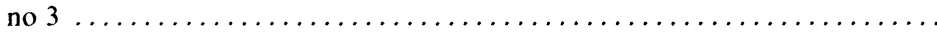

Canada et aux États-Unis, le chômage chez les hommes et les femmes au, par P.A. Della Valle et B. Meyer, no $3 \ldots \ldots \ldots \ldots \ldots \ldots \ldots \ldots \ldots$ Canada, la main-d'euvre hautement qualifiée au, par Avigdor Farine, no $2 . .$. . Canada, Highly Qualified Manpower in, by Avigdor Farine, no $2 . . . \ldots \ldots \ldots$ Canadian Approach, Public Sector Dispute Resolution: an American Twist to a,

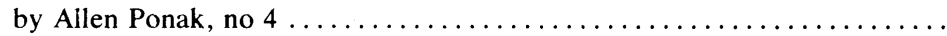

Canadian-United States Comparison, Changes in Relative Female-Male Unemployment : $a$, by P.A. Della Valle et B. Meyer, no $3 \ldots \ldots \ldots \ldots$. Canadian Anti-Inflation Board, the, by Claude Castonguay, no $4 \ldots \ldots \ldots \ldots$.

$366-367$

282-284 
Canadien de lutte à l'inflation, le programme, par Claude Castonguay, no 4 .... Changes in Relative Female-Male Unemployment: a Canadian-United States Comparison, by P.A. Della Valle et B. Meyer, no $3 \ldots \ldots \ldots \ldots \ldots \ldots$

Chômage et vacance d'emploi, par M.L. Skolnik et F. Siddiqui, no $1 \ldots \ldots \ldots$. Chômage et offre de travail, assurance-chômage, durée du, par Dennis $\mathrm{R}$.

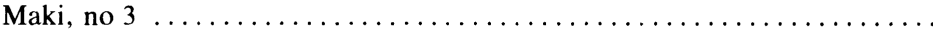

Chômage chez les hommes et les femmes au Canada et aux États-Unis, par P.A. Della Valle et B. Meyer, no $3 \ldots \ldots \ldots \ldots \ldots \ldots \ldots \ldots \ldots \ldots$

Chômage frictionnel, recherche d'emploi et, par C.J. Bruce et J.H. Marshall,

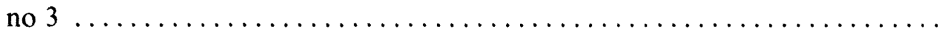

Communauté européenne et les législations nationales du travail, par $\mathrm{R}$.

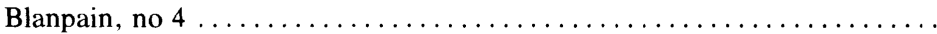

Communications dans une organisation: une étude de cas dans un hôpital, les,

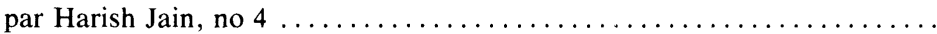

Communication: a Case Study of a Large Urban Hospital, Organizational,

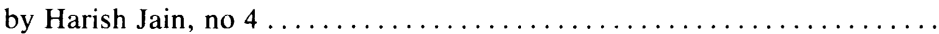

Company Satisfaction, Company Commitment and Work Involvement: An Empirical Examination of Blue Collar, by Vishwanath Baba et Muhammad

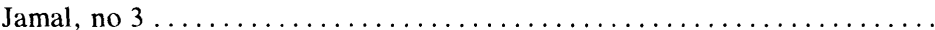

Comparison of Wage Determination Methods in the Americas, by Chris Jec-

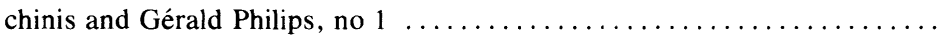

Complémentarité des approches systémique et scientifique dans le domaine des sciences humaines, par Maurice Landry et Jean-Louis Maloin, no 3....

Complementarity of the Scientific and Systemic Approaches in Human Sciences, by Maurice Landry and Jean-Louis Maloin, no $3 \ldots \ldots \ldots \ldots \ldots$

Conflits d'intérêts dans le secteur public, par Allen Ponak, no $4 \ldots \ldots \ldots \ldots$. . .

Construction Industry, A Note on Industrial Conflict in British Columbia's,

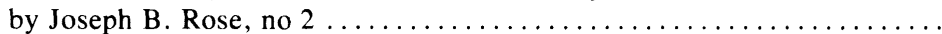

Cost/Benefit Analysis of a Strike: Theory and Application, by S.M. A. Hameed,

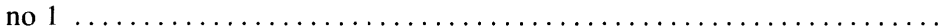

CTCC: une réponse à Louis-Marie Tremblay, l'idéologie initiale de la, par

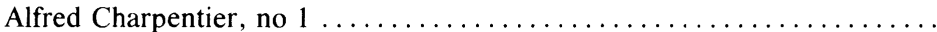

Current Status of Labour Legislation in Québec: 1968-1976, by Jean Bernier,

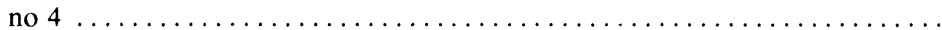

Decision Making, Faculty Unionism and Collegial, by Roy J. Adams, no 3.....

Dispute Resolution: an American Twist to a Canadian Approach, Public

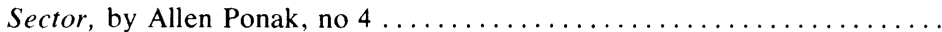

Economic Activity, Inflation and Strikes, by J.M. Cousineau and Robert Lacroix, no 3

Economic Activity: An Empirical Analysis, Strike Settlement and, by R.

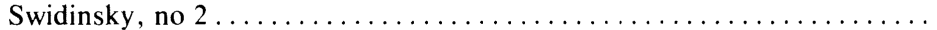

Emplois et la fixation des salaires dans le secteur public en Israël, l'évolution

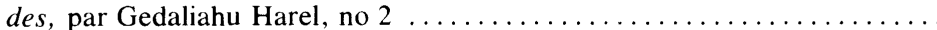

Engagement et participation, satisfaction, par V. Baba et M. Jamal, no 3 .... États-Unis, le chômage chez les hommes et les femmes au Canada et aux,

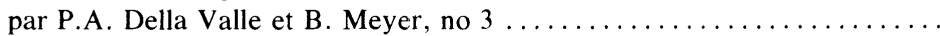

Étude comparative des méthodes de fixation des salaires dans les Amériques, de Chris Jecchinis et G. rald Phillips, no $1 \ldots \ldots \ldots \ldots \ldots \ldots \ldots \ldots \ldots$

Évaluation des emplois et la fixation des salaires dans le secteur public en

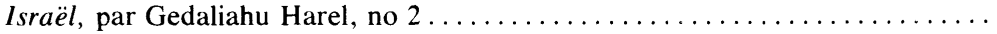


Évolution de la différence de salaire entre hommes et femmes en Ontario de 1946 à 1971, l', par Morley Gunderson, no $1 \ldots \ldots \ldots \ldots \ldots \ldots \ldots \ldots$

European Economic Community on National Labour Law Systems, The Impact of Recent Developments in the, by R. Blanpain, no $4 \ldots \ldots \ldots \ldots \ldots$

Faculty Unionism and Collegial Decision-Making, by Roy J. Adams, no 3....

Female Managers, Stimulating Recruitment of, par Maurice D. Levi, no 1.....

Female-Male Unemployment: a Canadian-United States Comparison, Changes in Relative, by P.A. Della Valle and B. Meyer, no $3 \ldots \ldots \ldots \ldots \ldots$

Femmes dans les postes administratifs, des moyens de stimuler le recrutement

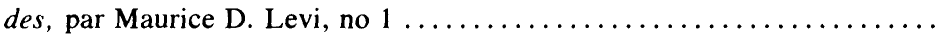

Femmes en Ontario de 1946 à 1971, l'évolution de la différence de salaire entre homme et, par Morley Gunderson, no $1 \ldots \ldots \ldots \ldots \ldots \ldots \ldots \ldots \ldots . \ldots \ldots$

Femmes au Canada et aux États-Unis, le chômage chez les hommes et les,

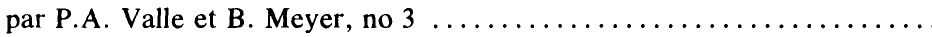

Frictional Unemployment: Some Empirical Evidence, Job Search and, by C.J.

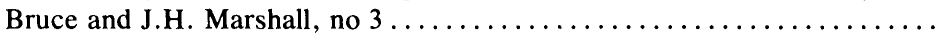

Full Employment and Social Policies : a European Lesson, by Klaus Weiermair, no 2

Full Employment and Social Policies: a European Lesson, by Klaus Weiermair, no 2 .

Gestion $d u$ stress, par Hans Seley et Pierre Turcotte, no $4 \ldots \ldots \ldots \ldots \ldots \ldots$

Grève, activité économique, inflation et activité de, par J.M. Cousineau et

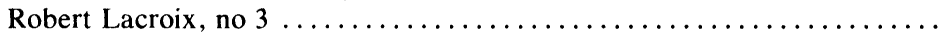

Grèves et l'activité économique, le règlement des, par $\mathrm{R}$. Swidinsky, no $2 \ldots \ldots$.

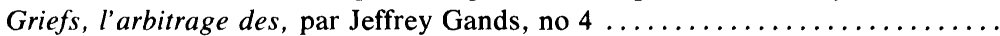

Grievance Arbitration. A Model for the Study of Policy Change, by Jeffrey

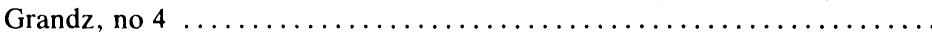

Highly Qualified Manpower in Canada, by Avigdor Farine, no $2 \ldots \ldots \ldots \ldots$

Hopital, les communications dans une organisation: une étude de cas dans un,

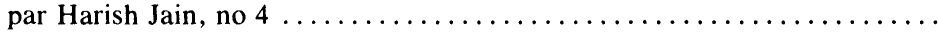

Hommes et femmes en Ontario de 1946 à 1971, l'évolution de la différence de salaire entre, par Morley Gunderson, no $1 \ldots \ldots \ldots \ldots \ldots \ldots \ldots \ldots$

Hospital, Organization Communication: A Case Study of a Large Urban

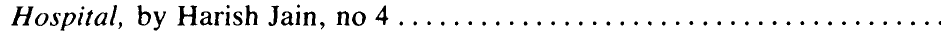

Human Sciences, The Complementarity of the Scientific and Systemic Approaches in, by Maurice Landry and Jean-Louis Maloin, no $3 \ldots \ldots \ldots$. Identifying Sources of Imbalance in Individual Labour Markets, by Noah

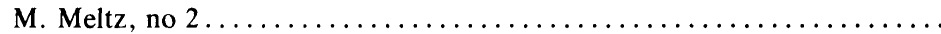

Idéologie initiale de la CTCC: une réponse à Louis-Marie Tremblay, par Alfred

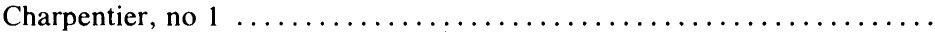

Impact of Inflation on Strike Activity in Canada, by Douglas A. Smith, no 1.... Impact of Recent Developments in the European Economic Community on National Labour Law Systems, by R. Blanpain, no 4 ............... Industrial Relations: Public and Private Sectors, Managerial Attitudes Toward, by Mark J. Thompson and Larry F. Moore, no $3 \ldots \ldots \ldots \ldots \ldots \ldots$ Industrial Relations, Theoretical Problems of Public Interest Sector, by Paul

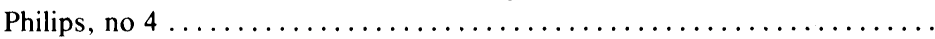
Industrial Conflict in British Columbia's, Construction Industry, a Note on,

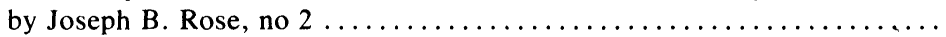
Inflation on Strike Activity in Canada, The Impact of, by Douglas A. Smith, no 1 
Inflation and Strikes, Economic Activity, by J.M. Cousineau and Robert Lacroix,

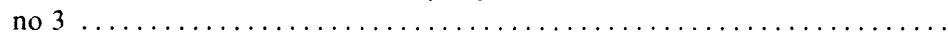

Inflation et activité de grève, activité économique, par J.M. Cousineau et

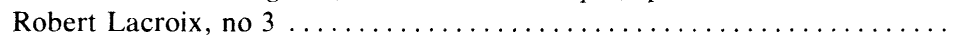

Inflation, Statics and Dynamics of, by E.F. Beach, no $4 \ldots \ldots \ldots \ldots \ldots$ Inflation, note sur la protection des salaires contre l', par Bertrand Belzile,

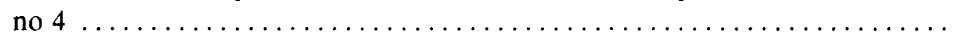

Inflation, le programme canadien de lutte à $l$ ', par Claude Castonguay, no $4 \ldots$. Intérêts dans le secteur public, les conflits d', par Allen Ponak, no $4 \ldots \ldots \ldots$. Intérêt public, problèmes théoriques des relations du travail dans les services

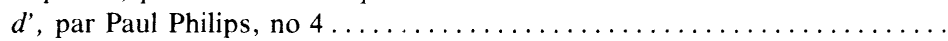

Israël, Job Evaluation and Wage Setting in the Public Sector of, by Gedaliahu

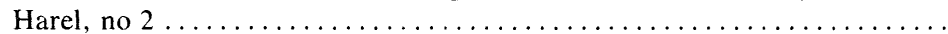

Israël, structure syndicale et révolte de la base: l'expérience d', par Abraham

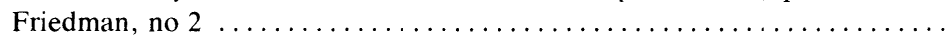

Israeli Experience, Union Structure and Rank and File Revolt: The, by Abraham Freidman, no 2

Israël, l'évaluation des emplois et la fixation des salaires dans le secteur public en, par Gedaliahu Harel, no 2

Job Search and Frictional Unemployment: Some Empirical Evidence, by C.J. Bruce and J.H. Marshall, no 3

Job Search and Wage Behaviour, par Claude Rondeau, no 2

Job Evaluation and Wage Setting in the Public Sector of Israël, by Gedaliahu Harel, no 2 ...

Kenya, On the Lack of Trade Union Power in, by John S. Henley, no $4 \ldots \ldots$.

Labour Legislation in Québec: 1968-1976, Current Status of, by Jean Bernier.

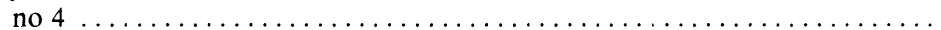

Labour Law Systems, The Impact of Recent Developments in the European Economic Community on National, by R. Blanpain, no $4 \ldots \ldots \ldots \ldots$.

Labour Markets, Identifying Sources of Imbalance in Individual, by Noah

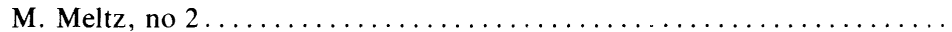

Lack of Trade Union Power in Kenya, by John S. Henley, no $4 \ldots \ldots \ldots \ldots$. . . .

Législations nationales du travail, la Communauté économique européenne et

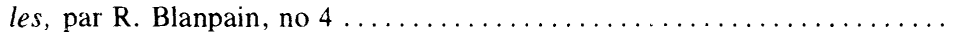

Législation québécoise en matière de relations du travail: 1968-1976, par Jean

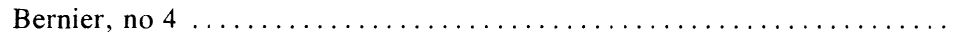

Licenciement collectif: l'expérience québécoise, préavis de, par Jean Sexton et

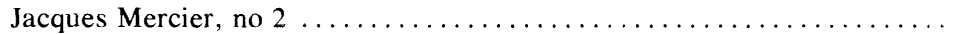
Local Unionization adn Social Action: Case Analysis, by L.H.J. Groulx, no 1 . . Main-d'euvre hautement qualifiée au Canada, par Avigdor Farine, no 3 ...... Management et prévention des accidents du travail - Les responsabilités des cadres à l'égard de la prévention, par Gérard Hébert, no $1 \ldots \ldots \ldots \ldots$.

Management, Some Aspects of Power and Independence in, by J.J. Howard,

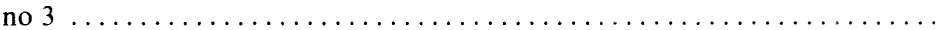

Managerial Attitudes Toward Industrial Relations: Public and Private Sectors, by Mark J. Thompson and Larry F. Moore, no $3 \ldots \ldots \ldots \ldots \ldots \ldots$

Management and Work Accident Prevention, by Gérard Hébert, no $1 \ldots \ldots \ldots$ Managers, Stimulating Recruitment of Female, by Maurice D. Levi, no 1..... Manpower in Canada, Highly Qualified, by Avidgor Farine, no $2 \ldots \ldots \ldots \ldots$. Marchés locaux du travail, sources de déséquilibre dans les, par Noah M.

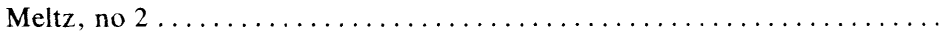


Mass Layoffs: the Québec Experience, Advance Notice of, by Jean Sexton et

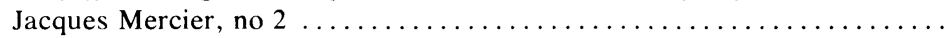

Moyens de stimuler le recrutement des femmes dans les postes administratifs,

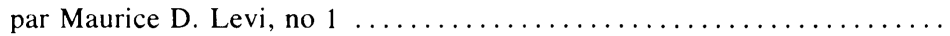

New Challenges of the, Office de la construction du Québec:, the, by R.

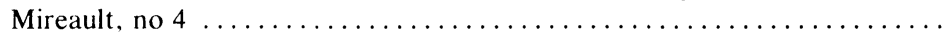

Note on Industrial Conflict in British Columbia's Construction Industry, by

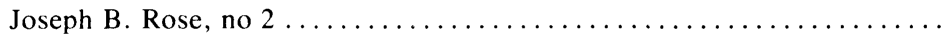

Note sur la protection des salaires contre l'inflation, par Bertrand Belzile, no 4 .

Nouveaux défis de l'Office de la construction du Québec, par R. Mireault, no 4.

Offre de travail, assurance-chômage, durée du chômage et, par Dennis R. Jaki,

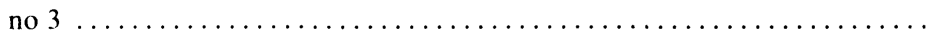

Ontario de 1946 à 1971, l'évolution de la différence de salaire entre hommes et femmes en, de Morley Gunderson, no $1 \ldots \ldots \ldots \ldots \ldots \ldots \ldots \ldots \ldots \ldots \ldots$

Organizational Communication: A Case Study of a Large Urban Hospital,

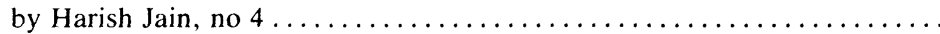

Paradox of Unemployment and Job Vacancies: Some Theories Confronted by

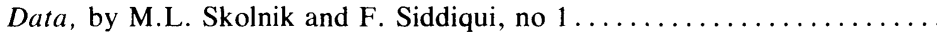

Participation and Industrial Relations - The trend Toward Decentralization, Worker, by Edward G. Wertheim, no $1 \ldots \ldots \ldots \ldots \ldots \ldots \ldots \ldots \ldots$

Participation des travailleurs et les régimes de relations industrielles, de

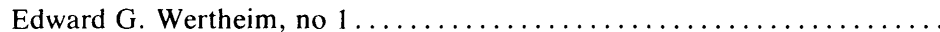

Participation, satisfaction, engagement et, par V. Baba et M. Jamal, no $3 \ldots .$.

Patronales en matière de relations professionnelles: secteurs public et privé, Attitudes, par Mark J. Thompson et Larry F. Moore, no 3 ............

Postes administratifs, des moyens de stimuler le recrutement des femmes dans

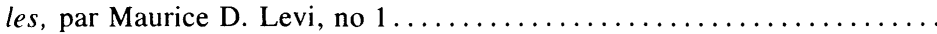

Power and Independence in Management, Some Aspects of, by J.J. Howard,

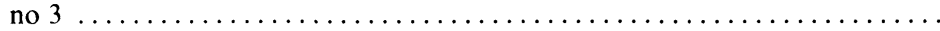

Préavis de licenciement collectif: l'expérience québécoise, par Jean Sexton et

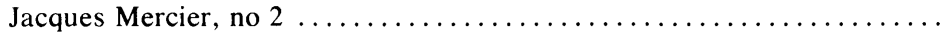

Prévention des accidents du travail, Management et - Les responsabilités des cadres à l'égard de la prévention, par Gérard Hébert, no $1 \ldots \ldots \ldots \ldots$.

Problèmes théoriques des relations du travail dans les services d'intérêt public,

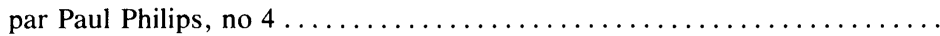

Programme canadien de lutte à l'inflation, par Claude Castonguay, no 4 .....

Public Interest Sector Industrial Relations Theoretical Problems of, by Paul

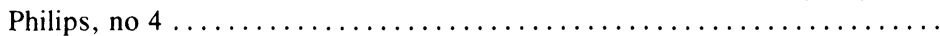

Public Sector Labor Relations in Sweden, by Gilles Coulombe, no 3 .........

Public Sector of Israël, Job Evaluation and Wage Setting in the, by Gedaliahu

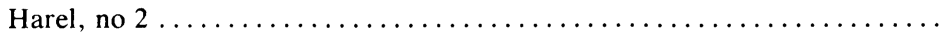

Public Sector Dispute Resolution: An American Twist to a Canadian Approach,

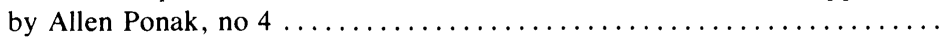

Québec: 1968-1976, Current Status of Labour Legislation in, by Jean Bernier,

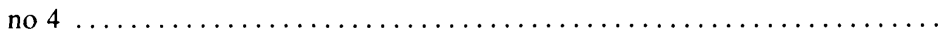

Québec Experience, Advance Notice of Mass Layoffs: The, by Jean Sexton et

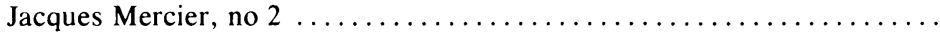

Québécoise en matière de relations du travail: 1968-1976, la législation, par

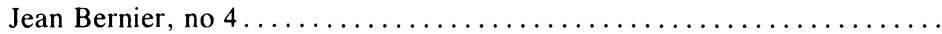

Québécoise, préavis de licenciement collectif: l'expérience, par Jean Sexton et Jacques Mercier, no 2 
Rank and File Revolt: The Israeli Experience, Union Structure and, by

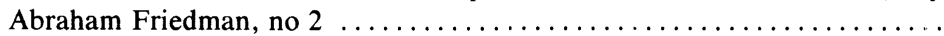

Recherche d'emploi et chômage frictionnel, par C.J. Bruce and J.H. Marshall,

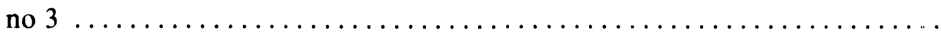

Recruitment of Female Managers, Stimulating, by Maurice D. Levi, no $1 . . .$. .

Recrutement des femmes dans les postes administratifs, des moyens de stimuler

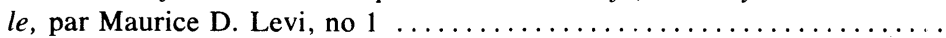

Règlement des grèves et l'activité économique, les, par R. Swidinsky, no $2 \ldots$.

Relations de travail dans le secteur public. Le modèle suédois: mythe ou réalité,

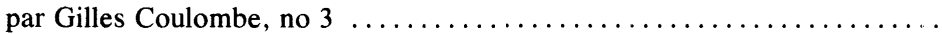

Relations industrielles, la participation des travailleurs et les régimes de, de

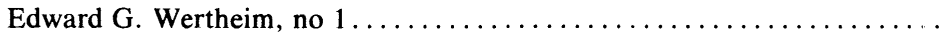

Relations professionnelles: secteur public et privé, attitudes patronales en matière de, par Mark J. Thompson et Larry F. Moore, no $3 \ldots \ldots \ldots \ldots \ldots$

Relations du travail dans les services d'intérêt public, problèmes théoriques des,

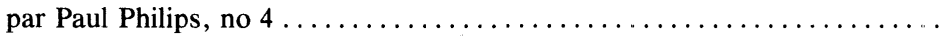

Relations du travail: 1968-1976, la législation québécoise en matière de, par

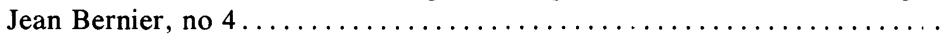

Salaire entre hommes et femmes en Ontario de 1946 à 1971, l'évolution de la différence de, par Morley Gunderson, no $1 \ldots \ldots \ldots \ldots \ldots \ldots \ldots$

Salaires dans les Amériques, Étude comparative des méthodes de fixation des, par Chris Jecchinis et Gérald Phillips, no $1 \ldots \ldots \ldots \ldots \ldots \ldots \ldots \ldots$

Salaires contre l'inflation, note sur la protection des, par Bertrand Belzile, no 4.

Salaires dans le secteur public en Israël, l'évaluation des emplois et la fixation

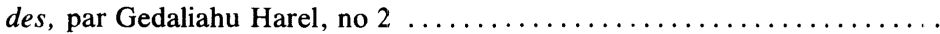

Satisfaction, engagement et participation, par V. Baba et M. Jamal, no 3 .... Satisfaction, Company Commitment and Work Involvement: An Empirical Examination of Blue Collar, by V. Baba and M. Jamal, no $3 \ldots \ldots \ldots \ldots$

Sciences humaines, La complémentarité des approches systémique et scientifique dans le domaine des, par Maurice Landry et Jean-Louis Maloin,

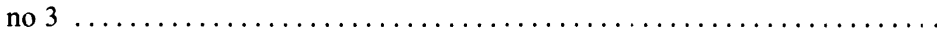

Scientific and Systemic Approaches in Human Sciences, the Complementarity of the, by Maurice Landry and Jean-Louis Maloin, no $3 \ldots \ldots \ldots \ldots \ldots$

Secteur public, les conflits d'intérêts dans le, par Allen Ponak, no 4 .........

Secteur public. Le modèle suédois: mythe ou réalité, relations de travail dans

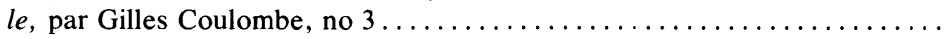

Secteur public en Israël, l'évaluation des emplois et la fixation des salaires dans le, par Gedaliahu Harel, no $2 \ldots \ldots \ldots \ldots \ldots \ldots \ldots \ldots \ldots \ldots \ldots \ldots . \ldots \ldots$

Services d'intérêt public, problèmes théoriques des relations du travail dans les,

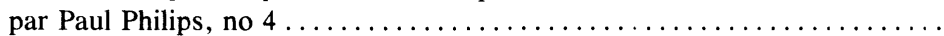

Social Action: Case Analysis, Local Unionization and, by L.H.J. Groulx,

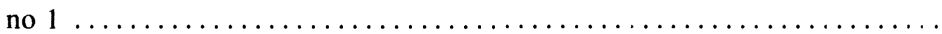

Some Aspects of Power and Independence in Management, by J.J. Howard,

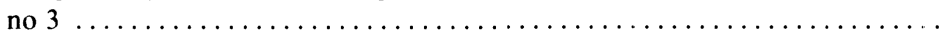

Sources de déséquilibre dans les marchés locaux du travail, par Noah M. Meltz,

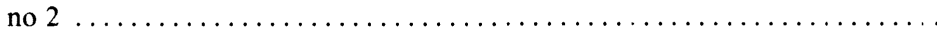

Statics and Dynamics of Inflation, by E.F. Beach, no $4 \ldots \ldots \ldots \ldots \ldots \ldots$

Stimulating Recruitment of Female Managers, by Maurice D. Levi, no 1 .....

Stress, la gestion $d u$, par Hans Seley et Pierre Turcotte, no $4 \ldots \ldots \ldots \ldots \ldots$. . . .

Strike: Theory and Application, Cost/Benefit Analysis of a, by S.M.A. Hameed, no 1

$366-367$

$587-588$

119-122

668-676

300-303

442-444

431-442

$379-400$

400-401

551-553

445-461

300-303

$587-588$

97-98

463-472

246- 247

676-682

72-82

609-617

145-156 
Strike Activity in Canada, The Impact of Inflation on, by Douglas A. Smith,

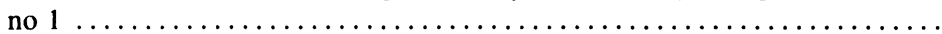

Strikes, Economic Activity, Inflation and, by J.M. Cousineau and Robert

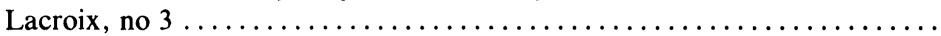

Strike Settlement and Economic Activity: An Empirical Analysis, by $\mathrm{R}$.

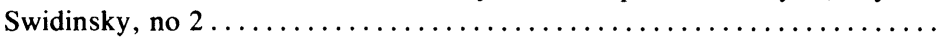

Structure syndicale et révolte de la base: l'expérience d'Israël, par Abraham

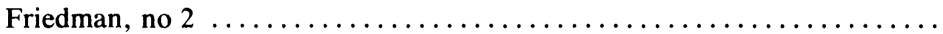

Suédois: mythe ou réalité, relations de travail dans le secteur public. Le

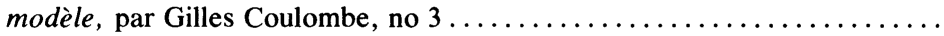

Supply of Labour, Unemployment Insurance Unemployment Duration, and

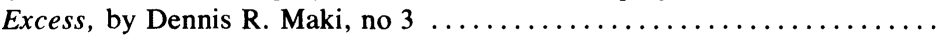

Sweden, Public Sector Labor Relations in, by Gilles Coulombe, no 3 ........

Syndicalisation locale et action ouvrière: analyse de cas, par L.H.J. Groulx,

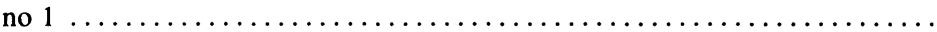

Technological Unemployment $-A$ Failure in Theorizing, by Earl F. Beach,

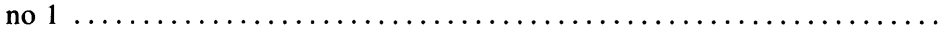

Theoretical Problems of Public Interest Sector Industrial Relations, by Paul

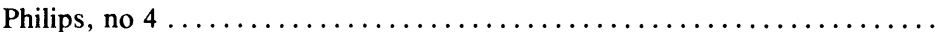

Time Pattern of Male-Female Wage Differentials: Ontario 1946-71, by Morley

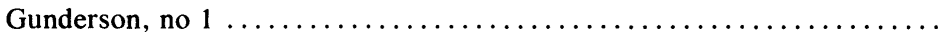

Trade Union Growth in Canada: a Comment, by George Bain et F. Elsheikh,

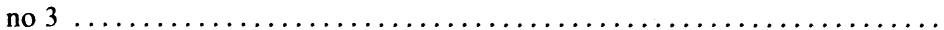

Trade Union Power in Kenya, On the Lack of, by John S. Henley, no 4 ......

Travailleurs et les régimes de relations industrielles, la participation des,

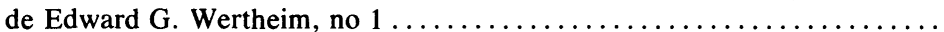

Unemployment : a Canadian-United States Comparison, Changes in Relative Female-Male, by P.A. Della Valle and B. Meyer, no $3 \ldots \ldots \ldots \ldots \ldots$

Unemployment - a Failure in Theorizing, Technological, by Earl F. Beach,

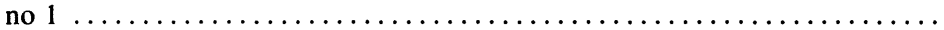

Unemployment: Some Empirical Evidence, Job Search and Frictional, by C.J. Bruce and J.H. Marshall, no $3 \ldots \ldots \ldots \ldots \ldots \ldots \ldots \ldots$

Unemployment Insurance, Unemployment Duration, and Excess Supply of Labour, by Dennis R, Maki, no $3 \ldots \ldots \ldots \ldots \ldots \ldots \ldots \ldots \ldots \ldots$

Union Structure and Rank and File Revolt: The Israeli Experience, by Abraham

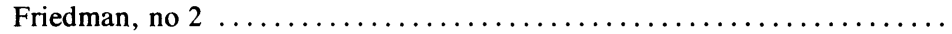

Unionism and Collegial Decision-Making, Faculty, by Roy J. Adams, no 3 ..... Vacance d'emploi, Chômage et, par M.L. Skolnik et F. Siddiqui, no $1 \ldots \ldots \ldots$ Wage Determination Methods in the Americas, a Comparison of, by Chris

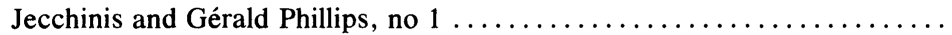

Wage Behaviour, Job Search and, by Claude Rondeau, no $2 \ldots \ldots \ldots \ldots \ldots$ Wage Setting in the Public Sector of Israël. Job Evaluation and, by Gedaliahu

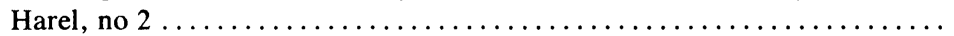
Work Accident Prevention, Management and, by Gérard Hébert, no $1 \ldots \ldots$. Work Involvement: An Empirical Examination of Blue Collar, Company Satisfaction, Company Commitment and, by V. Baba et M. Jamal, no 3 ... Worker Participation and Industrial Relations - The Trend Toward Decentralisation, by Edward G. Wertheim, no $1 \ldots \ldots \ldots \ldots \ldots \ldots \ldots$ 


\section{Auteurs des ouvrages recencés}

BAKER, Frank, Organizational Systems, General Systems Approaches to Complete organization, (Laurent Bélanger), no $2 \ldots \ldots \ldots \ldots \ldots \ldots$

BATTEN, J. D. Direction par les objectifs et motivation des hommes, (Laurent Bélanger), no 3

BEAUCAGE, André, Brève description du système des relations du travail

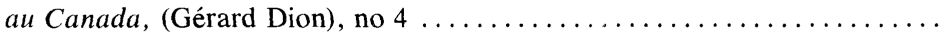

BECKARD, Richard, Le développement des organisations stratégies et modèles,

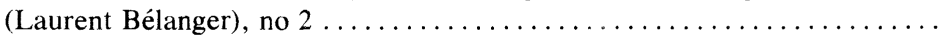

BENNIS, Warren G., Le développement des organisations: sa pratique, ses perspectives et ses problèmes, (Laurent Bélanger), no $2 \ldots \ldots \ldots \ldots \ldots$

BRUN, André, Études de droit de travail, (Rodrigue Blouin), no $1 \ldots \ldots \ldots \ldots$.

BRUNELLE, D. Le Code civil et les rapports de classes, (Rodrigue Blouin),

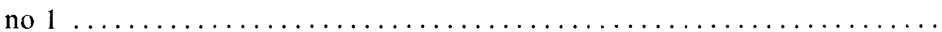

COHER, Sanford, Labor in the United States (Hem C. Jain), no 1 ..........

CONFÉRENCE INTERNATIONALE DU TRAVAIL, Liberté syndicale et procédure de détermination des conditions d'emploi dans la fonction publique, Rapport VII, (Rodrigue Blouin), no $3 \ldots \ldots \ldots \ldots \ldots \ldots \ldots$

CONNAGHAN, Charles, Partnership or Marriage of Convenience?, (Ray

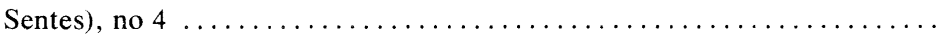

CONSEIL ÉCONOMIQUE DU CANADA, Des travailleurs et des emplois, (Émilien Landry), no 4

$324-325$

687

$322-323$

$322-323$

$157-158$

157

$162-164$

$491-493$

$682-684$

$687-689$

CUTLER, Philip, Code du travail du Québec, Édition revisée, (Gérard Dion), no 3

DAVIS, Louis E. and Albert B. CHERNS (éd.), The Quality of Working Life. Vol. I: «Problems, Prospects and the state of the Art», Vol. II: «Cases and Commentary», (Gilles Dussault), no $2 \ldots \ldots \ldots \ldots \ldots \ldots \ldots \ldots$

DESGAGNE, André et Roger MILLER, L'Université et la syndicalisation de ses professeurs, (Gilles Dussault), no $1 \ldots \ldots \ldots \ldots \ldots \ldots \ldots \ldots \ldots \ldots \ldots \ldots \ldots \ldots$

DESPAX, M. et PELLISSIER, J., La gestion du personnel: aspects juridiques,

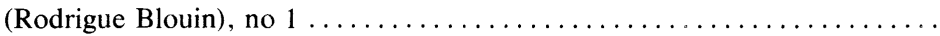

DOSSIERS DE QUÉBEC-SCIENCE, Demain la santé, (Gilles Dussault), no 2.

FISHER, Malcolm, Mesure des conflits du travail et de leurs répercussions économiques, (Jean Bernard Fournier), no $1 \ldots \ldots \ldots \ldots \ldots \ldots \ldots \ldots \ldots$

JURIS, Hervey A, and Peter FEUILLE, Police Unionism: Power and Impact in Public-Sector Bargaining, (Jacques Bélanger), no $4 \ldots \ldots \ldots \ldots \ldots \ldots$

KAY, Emmanuel, Cadres: la cote d'alerte, (Laurent Bélanger), no 2 .........

KURILOFF, Arthur, Organizational Development for Survival, (Laurent Bélan-

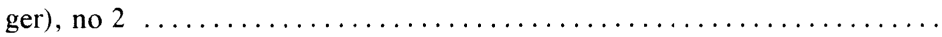

MALLES, Paul, Insécurité d'emploi et relations de travail dans l'industrie canadienne de la construction, (Jean Bernard Fournier), no $1 \ldots \ldots \ldots \ldots$

MANCUSO, Thomas F., Help for the Working Wounded, (Ray Sentes), no 4...

OFFICE DES PROFESSIONS du Québec, L'évolution du professionnalisme,

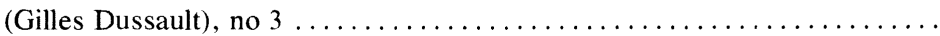

PEITCHINIS, Stephen B., The Canadian Labour Market, (Bertrand Belzile),

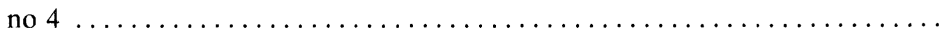

REHMUS, Charles M., Public Employment Labor Relations: An overview of Eleven Nations, (Jean Boivin), no 1

$326-327$

156-157

164

497-498

159-160

689-690

322

323-324

161-162

684-685

685-687

159-160 
REPORT OF THE MANITOBA ECONOMIC DEVELOPMENT ADVISORY

BOARD, Manpower Issues in Manitoba, (Jean Sexton), no $1 \ldots \ldots \ldots \ldots$

RINEHART, James W., The Tyranny of Work, (Gilles Dussault), no 2 .......

ROTSTEIN, Abraham, Beyond Industrial Growth, (Gilles Dussault), no 2 ....

$160-161$

$325-326$

326

SELLIER, François, Les relations industrielles: principes et politiques, (Roger

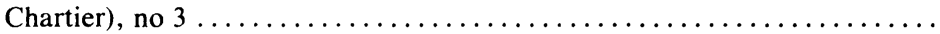

TRAVAIL-CANADA, Industrial Relations Research in Canada/La recherche sur les relations industrielles au Canada, (Gérard Dion), no $4 \ldots . . . . . .$.

WOOD, W. D., The Current Trade Union Scene, Patterns and Trends, (Gérard Dion), no 4

$493-495$

682

687

\section{Auteurs d'articles}

ADAMS, Roy, J., Faculty Unionism and Collegial Decision-Making, no 3.....

BABA, Vishwanath and JAMAL, Muhammad, Company satisfaction, Company Commitment and Work Involvement: An Empirical Examination of

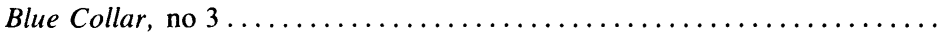

BABA, Vishwanath and Muhammad JAMAL, Company Satisfaction, Company Commitment and Work Involvement: An Empirical Examination of Blue

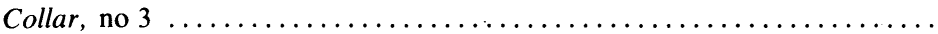

BAIN, George and F. ELSHEIKH, Trade Union Growth in Canada: A Com-

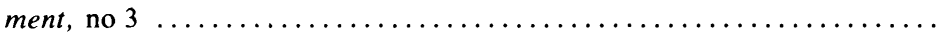

BAIN, George and F. ELSHEIKH, Trade Union Growth in Canada: A Com-

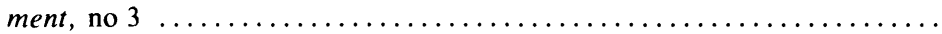

BEACH, Earl F. Technological Unemployment $-A$ Failure in Theorizing,

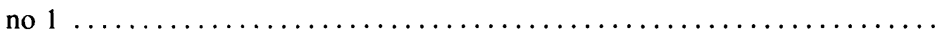

BEACH, E. F., Statics and Dynamics of Inflation, no $4 \ldots \ldots \ldots \ldots \ldots \ldots$

BELZILE, Bertrand, Note sur la protection des salaires contre l'inflation, no 4.

BERNIER, Jean, La législation québécoise en matière de relations du travail:

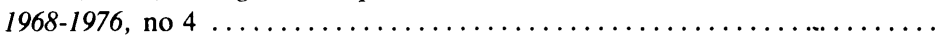

BLANPAIN, R., The Impact of Recent Developments in the European Economic Community on National Labour Law Systems, no 4 ............

BRUCE, C. J. and J. H. MARSHALL, Job Search and Frictional Unemployment: Some Empirical Evidence, no $3 \ldots \ldots \ldots \ldots \ldots \ldots \ldots \ldots \ldots$

BRUCE, C. J. and J. H. MARSHALL, Job Search and Frictional Unemployment: Some Empirical Evidence, no $3 \ldots \ldots \ldots \ldots \ldots \ldots \ldots \ldots . . . \ldots \ldots$

CASTONGUAY, Claude, Le programme canadien de lutte à l'inflation, no $4 \ldots$

CHARPENTIER, Alfred, L'idéologie initiale de la CTCC: une réponse à

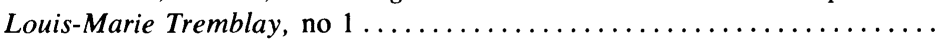

COULOMBE, Gilles, Relations de travail dans le secteur public. Le modèle

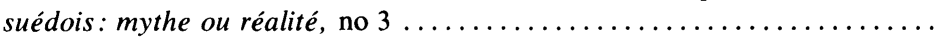

COUSINEAU, J. M. et Robert LACROIX, Activité économique, inflation et

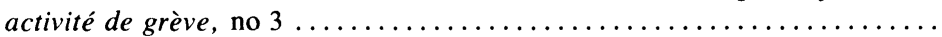

COUSINEAU, J. M. et Robert LACROIX, Activité économique, inflation et

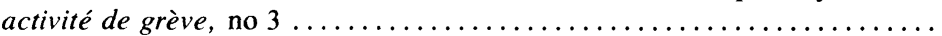

DELlA VALLE, P. A. and B. MEYER, Changes in Relative Female-Male Unemployment : a Canadian-United States Comparison, no 3 ..........

$476-482$

$434-445$

$434-445$

$482-491$

$482-491$

122-132

676-682

668-676

617-725

509-519

$402-415$

522-536

448-464

341-357

341-357

$417-431$ 
DELlA VALLE, P. A. and B. MEYER, Changes in Relative Female-Male Unemployment : a Canadian-United States Comparison, no $3 \ldots \ldots \ldots$.......

FERINE, Avigdor, La main-d' euvre hautement qualifiée au Canada, no 2 ..... FRIEDMAN, Abraham, Union Structure and Rank and File Revolt: The

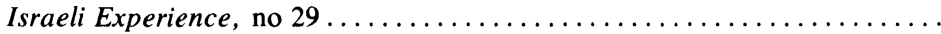

GANDZ, Jeffrey, Grievance Arbitration. A Model for the Study of Public

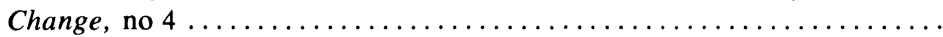

GROULX, L. H. J., Syndicalisation locale et action ouvrière: analyse de

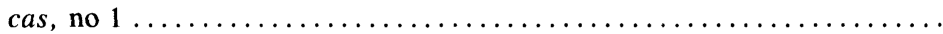

GUNDERSON, Morley, Time Pattern of Male-Female Wage Differentials:

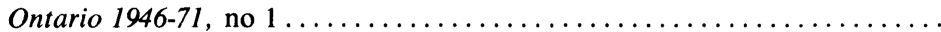

HAMEED, S. M. A., Cost/Benefit Analysis of a Strike: Theory and Applica-

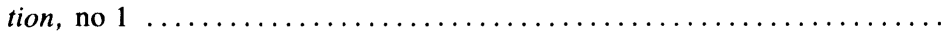

HAREL, Gedaliahu, Job Evaluation and Wage Setting in the Public Sector

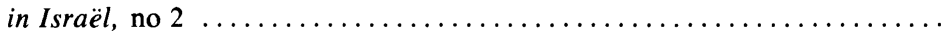

HÉBERT, Gérard, Management et prévention des accidents du travail - Les responsabilités des cadres à l'égard de la prévention, no $1 \ldots \ldots \ldots \ldots . . . . .$.

HENLEY, John S., On the Lack of Trade Union Power in Kenya, no 4 .......

HOWARD, J. J., Some Aspects of Power and Independence in Management,

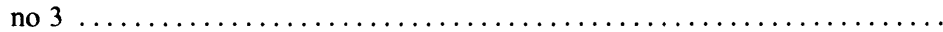

JAIN, Harish, Organizational Communication: A Case Study of a Large Urban

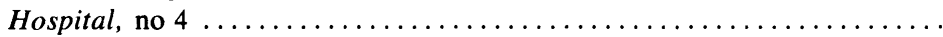

JECCHINIS, Chris and Gérald PHILliPS, A Comparison of Wage Deter-

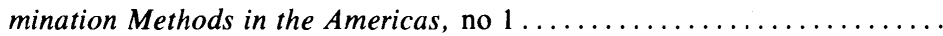

LANDRY, Maurice et Jean-Louis MALOIN, La complémentarité des approches systémique et scientifique dans le domaine des sciences humaines,

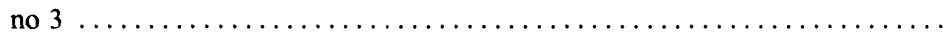

LANDRY, Maurice et Jean-Louis MALOUIN, La complémentairté des approches systémique et scientifique dans le domaine des sciences humaines,

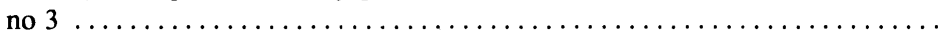

LEVI, Maurice D., Stimulating Recruitment of Female Managers, no 1 ...... MAKI, Dennis R., Unemployment Insurance, Unemployment Duration, and

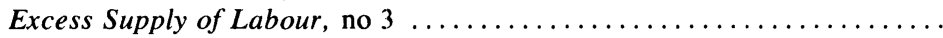

MELTZ, Noak M., Identifying Sources of Imbalance in Individual Labour

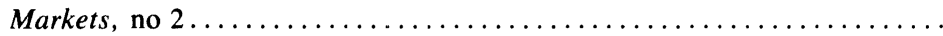

MERCIER, Jacques et Jean SEXTON, Préavis de licenciement collectif:

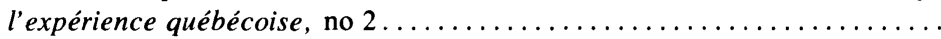

MIREAULT, R., Les nouveaux défis de l'Office de la construction du Québec,

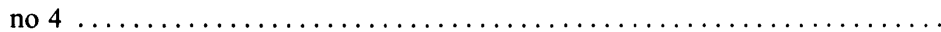

PHILLIPS, Gérald and Chris JECCHINIS, A Comparison of Wage Determina-

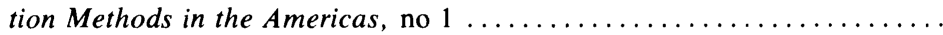

PHILIPS, Paul, Theoretical Problems of Public Interest Sector Industrial

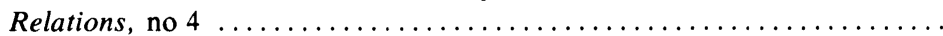

PONAK, Allen, Public Sextor Dispute Resolution: An American Twist to a

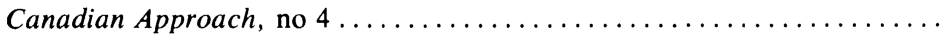

ROSE, Joseph B., A Note on Industrial Conflict in British Columbia's Construc-

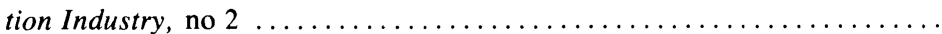

RONDEAU, Claude, Job Search and Wage Behaviour, no $2 \ldots \ldots \ldots \ldots \ldots$

SELYE, Hans et TURCOTTE, Pierre, La gestion $d u$ stress, no $4 \ldots \ldots \ldots \ldots$

SEXTON, Jean et Jacques MERCIER, Préavis de licenciement collectif:

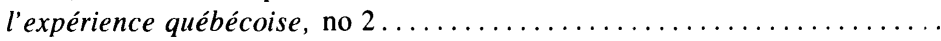


SIDDIQUI, F. and M. L. SKOLNIK, The Paradox of Unemployment and Job Vacancies: Some Theories Confronted by Data, no $1 \ldots \ldots \ldots \ldots$ SKOLNIK, M. L. and F. SIDDIQUI, The Paradox of Unemployment and Job Vacancies: Some Theories Confronted by Data, no $1 \ldots \ldots \ldots \ldots$

SMITH, Douglas A., The Impact of Inflation on Strike Activity in Canada, no 1.

SWIDINSKY, R., Strike Settlement and Economic Activity: An Empirical

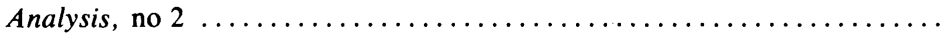

THOMPSON, Mark and Larry F. MOORE, Managerial Attitudes Toward

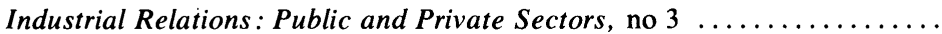

THOMPSON, Mark J. and Larry F. MOORE, Managerial Attitudes Toward Industrial Relations: Public and Private Sectors, no $3 \ldots \ldots \ldots \ldots$.

WEIERMAIR, Klans, Full Employment and Social Policies: A European

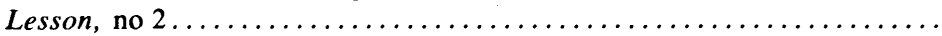

WERTHEIM, Edward G., Worker Participation and Industrial Relations - The French Toward Decentralization, no $1 \ldots \ldots \ldots \ldots \ldots \ldots \ldots \ldots \ldots$

\section{POUVOIR ET « POUVOIRS » EN RELATIONS DU TRAVAIL}

Introduction, Gérard Dion -- Pouvoir et «pouvoirs > dans les relations du travail, Vincent Lemieux et GÉRARD Dion - Pouvoir et e pouvoirs 》 dans l'entreprise privée. Hugues LeYdet - Pnuvoir et «pouvoirs 》 chez l'étatemployeur, JeAN COURnOYer - Pouvoir et «pouvoirs 》 dans les syndicats, Gérard Hébert - Pouvoir et action syndicale, Daniel Vidal - Pouvoir syndical, Bernard Solasse - Documents de travail.

\section{1 volume, 184 pages - Prix : $\$ 5.50$ \\ LES PRESSES DE L'UNIVERSITÉ LAVAL}

